\title{
Acute attack in a patient with multiple sclerosis 2 days after COVID vaccination: a case report
}

\author{
Maral Seyed Ahadi ${ }^{1} \cdot$ Fereshteh Ghadiri $^{1} \cdot$ Mohammad Ali ahraian $^{1} \cdot$ Abdorreza Naser Moghadasi $^{1} \mathbb{C}$
}

Received: 5 May 2021 / Accepted: 3 August 2021 / Published online: 11 August 2021

(c) Belgian Neurological Society 2021

\section{Introduction}

The effect of vaccination on multiple sclerosis (MS) has been debated since about 60 years ago when the first cases of relapses after inoculation were reported [1]. With novel vaccines introduced to tackle the recent pandemic, the issue is again highlighted. Primary reports show the safety of COVID-19 vaccination in MS patients [2]. Still, there is a possibility that the more people get vaccinated with various newly introduced vaccines, the more we may face unexpected adverse events. Here we report a relapse in a stable patient with MS 2 days after vaccination.

\section{Case report}

This is a report of a 42-year-old woman, a known case of relapsing-remitting MS diagnosed 20 years ago with optic neuritis. She was treated with Interferon beta-1a weekly and after a year escalated to interferon-beta $1 \mathrm{~b}$. After 2 years of treatment, she discontinued her treatment and only received intramuscular corticosteroid injections for each attack. She had two relapses 10 and 8 years ago manifested with hemiparesthesia and monoparesis, respectively. Her last attack was six years ago presented by paraparesis. She received her first dose of the Sinopharm vaccine (BBIBP-CorV's Beijing Institute of Biological Products) on April 13th, 2021. Two days later she noticed a progressive paraparesis without paresthesia and came to our MS clinic for the first time. On general examination, she did not have fever, constitutional or respiratory signs, and symptoms. Her blood oxygen saturation was $99 \%$. On neurologic examination, she had mild atrophic optic nerves on fundoscopy. Other cranial nerve

Abdorreza Naser Moghadasi

abdorrezamoghadasi@gmail.com

1 Multiple Sclerosis Research Center, Neuroscience Institute, Tehran University of Medical Sciences, Tehran, Iran examinations were unremarkable. On motor examination, her lower extremities' force was reduced to $4 / 5$ on the right and $3 / 5$ on the left side in hip flexion/extension, knee flexion/extension, foot dorsiflexion, and plantar flexion. Her deep tendon reflexes were brisk in the lower extremities and plantar reflexes were upward. Her sensory examinations were within normal limits. Cerebellar examination in the upper extremities showed bilateral intentional tremor. She was admitted and evaluated for attack and pseudo-attack. Her laboratory data disclosed normal erythrocyte sedimentation rate (ESR) and C-reactive protein (CRP), complete blood count (CBC), and urine analysis and culture. Her Brain and cervical magnetic resonance imaging (MRI) showed numeral periventricular, anterior temporal, cerebellar and anterior medullary white matter hyper-intensities (Fig. 1A-C). On gadolinium injection, there was a nodular enhancement in brainstem plaque, consistent with an active lesion of MS (Fig. 1D). She was treated with $1 \mathrm{~g} /$ day intravenous methylprednisolone for 5 days.

\section{Discussion}

Sufficient data have been available to assure the safety of vaccination against various diseases in MS patients [3, 4]. Now, facing an unexpected devastating pandemic, MS experts emphasize early vaccination against SARS-CoV2 in this population, although with some considerations [5]. Early reports do not show any adverse effect of the BNT162b2 COVID-19 vaccine (mRNA-based) on MS relapses [2]. This study showed that although some relapses happened in the days following vaccination, the rate was similar to the same periods in previous studies. But we found no report on other vaccines and their relation to relapse rate in MS patients. Of note, this coincidence does not imply any true association between the mentioned vaccine and MS relapse. However, it reemphasizes the importance of detailed documenting and further studies evaluating the association. 

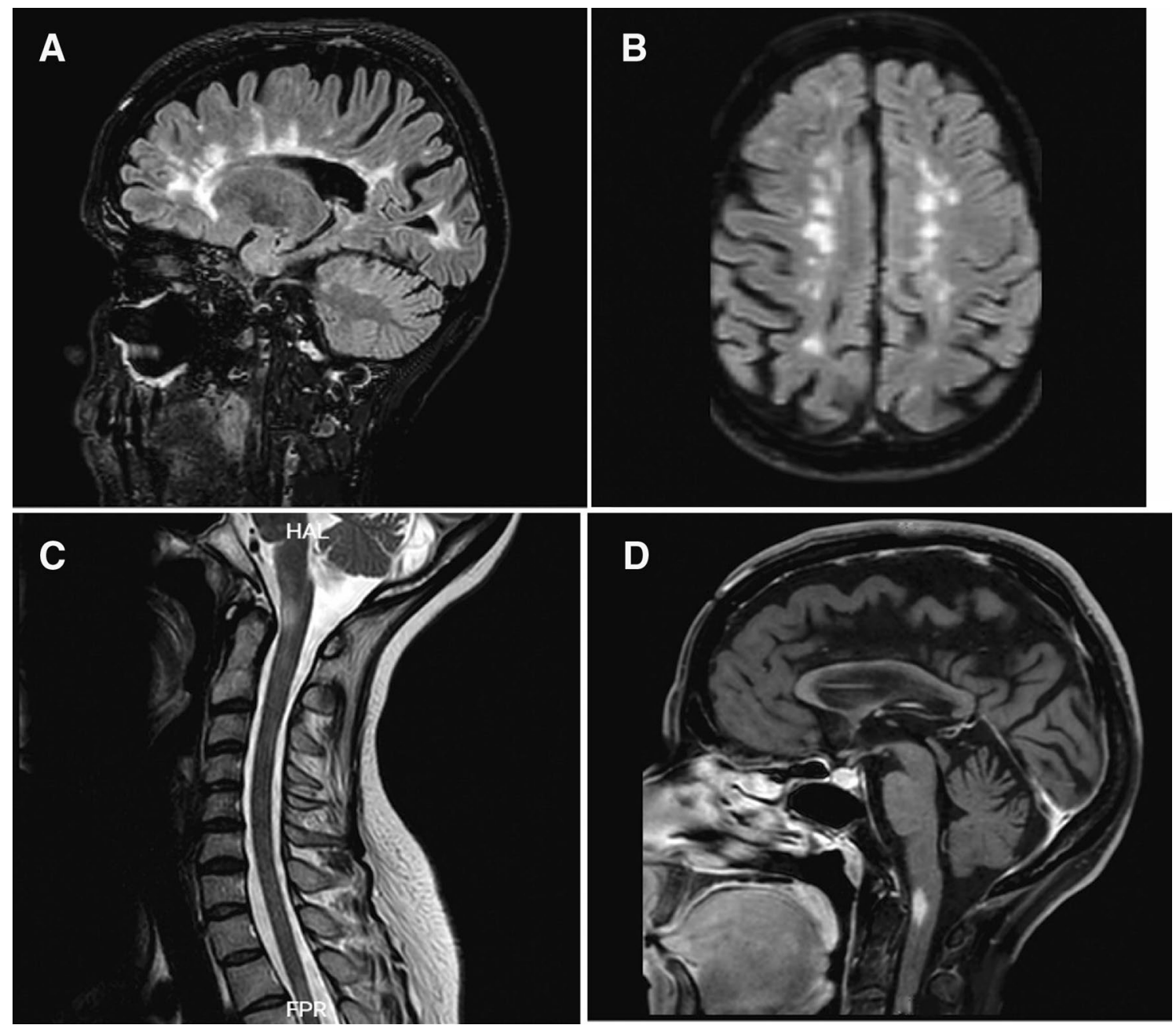

Fig. 1 A-C Brain and cervical MRI showed numeral periventricular, anterior temporal, cerebellar and anterior medullary white matter hyperintensities. D On gadolinium injection, there was a nodular enhancement in brainstem plaque, consistent with an active lesion of MS

\section{Declarations}

Conflict of interest The authors declare there is no conflict of interest.

Ethical approval All procedures performed in studies involving human participants were in accordance with the ethical standards of the institutional and/or national research committee and with the 1964 Helsinki declaration and its later amendments or comparable ethical standards.

Informed consent Informed consent was obtained from all participants.

\section{References:}

1. Miller H, Cendrowski W, Shapira K (1967) Multiple sclerosis and vaccination. Br Med J 2(5546):210-213
2. Achiron A, Dolev M, Menascu S, Zohar DN, Dreyer-Alster S, Miron S et al (2021) COVID-19 vaccination in patients with multiple sclerosis: What we have learnt by February 2021. Mult Scler 27(6):864-870

3. Mailand MT, Frederiksen JL (2017) Vaccines and multiple sclerosis: a systematic review. J Neurol 264(6):1035-1050

4. Farez MF, Correale J (2011) Immunizations and risk of multiple sclerosis: systematic review and meta-analysis. J Neurol 258(7):1197-1206

5. Otero-Romero S, Ascherio A, Lebrun-Frenay C (2021) Vaccinations in multiple sclerosis patients receiving disease-modifying drugs. Curr Opin Neurol 34(3):322-328

Publisher's Note Springer Nature remains neutral with regard to jurisdictional claims in published maps and institutional affiliations. 